\title{
A Syndrome of Vitamin D3 Deficiency/Fibromyalgia/Hyperparathyroidism Mimicking Rheumatoid Arthritis, a Clinical Prospective Study
}

\author{
Adel Elbeialy ${ }^{1^{*}(0)}$, Abdlnby Bauomi², Basma Elnaggar ${ }^{1}$, Hala Elzomor ${ }^{1}$ \\ ${ }^{1}$ Rheumatology, Departments Al-Azhar faculty of Medicine, Cairo, Egypt \\ ${ }^{2}$ Radiology, Departments Al-Azhar faculty of Medicine, Cairo, Egypt \\ Email: *aelbeialy@azhar.edu.eg
}

How to cite this paper: Elbeialy, A. Bauomi, A., Elnaggar, B. and Elzomor, H. (2021) A Syndrome of Vitamin D3 Deficiency/Fibromyalgia/Hyperparathyroidism Mimicking Rheumatoid Arthritis, a Clinical Prospective Study. Open Journal of Rheumatology and Autoimmune Diseases, $11,79-88$.

https://doi.org/10.4236/ojra.2021.112010

Received: April 21, 2021

Accepted: May 24, 2021

Published: May 27, 2021

Copyright ( 2021 by author(s) and Scientific Research Publishing Inc. This work is licensed under the Creative Commons Attribution International License (CC BY 4.0).

http://creativecommons.org/licenses/by/4.0/

\section{(c) (i) Open Access}

\begin{abstract}
Objectives: Rheumatoid arthritis is sometimes misdiagnosed for other diseases, like psoriatic arthritis, erosive OA, viral arthritis, reactive arthritis, IBD arthritis, Lyme's disease, and palindromic rheumatism. Secondary hyperparathyroidism was not included in the differential diagnosis of RA, though it sometimes presents with joint pains and tenderness, and even arthritis. Fibromyalgia is a psychosomatic disorder characterized by chronic widespread pain and tender areas. Mimicry of some manifestations of these diseases sometimes results in misdiagnosis as RA. Methods: Two hundred patients previously diagnosed as RA from outpatient clinics in Al-Azhar Faculty of Medicine, attended as not responding to medical treatment of RA. All patients were subjected to a re-evaluation of disease activity including HAQ, and DAS 28, CDAI, and SDAI. Also, we measured serum vitamin D3, PTH, total and ionized Calcium, Phosphorus, Uric acid, ACPA, and other routine lab. All patients were exposed to various radiological imaging modalities. Results: Cases not responding to RA treatment were reevaluated and were found to have a syndrome of fibromyalgia associated with vitamin D3 deficiency and secondary hyperparathyroidism. Conclusions: Fibromyalgia/Hyperparathyroidism syndrome is an underdiagnosed disease, which results from chronic vitamin D3 deficiency. SHPT can cause bone erosions, which are mostly shaggy in appearance and distributed in the radiocarpal, radioulnar, metacarpophalangeal and distal interphalangeal joints, in contrast to that which predominate proximal IP joints of rheumatoid arthritis. Radiology of FM/HPT syndrome patients revealed a sign of spur-like excrescences in terminal finger tufts unilaterally or bilaterally, which we think is pathognomonic.
\end{abstract}




\section{Keywords}

Secondary Hyperparathyroidism, Rheumatoid Arthritis, Vitamin D3, Cadmium Toxicity, Fibromyalgia

\section{Introduction}

Secondary hyperparathyroidism (SHPT) is the medical condition of excessive secretion of parathyroid hormone (PTH) by the parathyroid glands in response to hypocalcemia, with resultant hyperplasia of these glands. Chronic kidney failure is the most common cause of SHPT due to failure of kidneys to not convert enough vitamin D2 to its active form D3, and hence to not adequately excrete phosphate. When this happens, insoluble calcium phosphate forms in the body and removes calcium from the circulation. Both processes lead to hypocalcemia and hence SHPT [1]. SHPT can also result from malabsorption (chronic pancreatitis, small bowel disease, malabsorption-dependent bariatric surgery) in that the fat-soluble vitamin D cannot get reabsorbed. This leads to hypocalcemia and a subsequent increase in parathyroid hormone secretion in an attempt to increase the serum calcium levels. A few other causes can stem from inadequate dietary intake of calcium, a vitamin D deficiency, or steatorrhea [2].

HPT is usually associated with muscle cramps and bone pain, perioral tingling or paresthesia in fingers or toes, positive Chvostek and Trousseau signs. Arthritis simulating rheumatoid arthritis as the presenting feature of HPT was reported in literatures only rarely [3].

Rheumatoid arthritis (RA) is a chronic autoimmune disease that primarily affects joints. It typically results in warm, swollen, and painful joints. Most commonly, wrists and hands are involved, with the same joints typically involved bilaterally. It was known that RF is not specific for RA and could be found in other diseases such as Epstein-Barr virus or Parvovirus infections and in 5\% to $10 \%$ of healthy persons, especially the elderly [4] [5]. Anti-CCP antibodies are produced locally at sites of inflammation, not only in the synovium of RA, but also in other non-RA diseases. Therefore, anti-CCP positivity may be expected in a proportion of patients with non-RA diseases as well [6].

As we know, one of the causes of SHPT is vitamin D3 deficiency which was found to be widespread in Middle East whilst the area is rich in sun rays and consequently UV exposure. One of the most probable causes of vitamin D deficiency in Middle East area is pollution with heavy metals, like cadmium and lead [7] [8]. The mitochondria of proximal convoluted tubule cells of the kidney are the site of production of circulating 1a,25-dihydroxyvitamin D3 [1,25(OH)2D3], and parathyroid hormone $(\mathrm{PTH})$ markedly stimulates $1,25(\mathrm{OH}) 2 \mathrm{D} 3$ production, while 1,25(OH)2D3 suppresses production of PTH itself [9].

Cadmium preferentially selects to settle in kidneys. Renal cadmium deposition induces injury resulted from accumulation of cadmium-metallothionein (CdMT) 
in the proximal convoluted tubules. CdMT-induced nephrotoxicity might be due, at least in part, to its preferential uptake of CdMT into the S1 and S2 segments of the proximal tubules, which is responsible for converting 25(OH)D3 to $1,25(\mathrm{OH}) 2 \mathrm{D} 3[10]$.

Vitamin D3 deficiency and HPT are established to be associated with Fibromyalgia syndrome [11] [12]. Hyperparathyroidism is known to be associated with multiple articular manifestations including gout, pseudogout, juxtaarticular erosions, tenosynovitis, periarthritis, and pericapsular calcification [13].

\section{Patients and Methods}

This study included 200 female patients attended outpatient clinics of Al-Azhar University Faculty of medicine Hospitals, who had a previous diagnosis of RA and not responding to classic treatment including DMARDs, and had been allocated for biological therapy (Table 1).

\section{Inclusion and Exclusion Criteria}

All patients were diagnosed according to old and new criteria for classification of RA [14] [15], with exclusion of other mimicking diseases like psoriatic arthritis, erosive $\mathrm{OA}$, viral arthritis, reactive arthritis, IBD arthritis, Lyme's disease, and palindromic rheumatism.

Patients were reevaluated using old and new criteria for classification of RA. [14] [15]. We applied activity indices DAS 28, CDAI, and SDAI, [16] besides lab tests of RF, ACPA, ESR, CRP, LFT, RFT, serum vitamin D3, PTH, total and ionized calcium, phosphorus, and SUA. In addition, all patients did plain X-ray (Toshiba Digital Radiography System, DIGIX U) for hands, knees, and lumbar regions. When recommended, they were exposed to MRI (Philips1.5 tesla), and CT (Multi-Slice spiral CT Aston). High-resolution peripheral quantitative computed tomography (HR-pQCT) imaging allowed for highly reliable assessment of erosion in patients with rheumatoid arthritis (RA). As we noticed presence of fatigue and cognitive disorders in many patients, for that we applied old and new criteria for classification of fibromyalgia syndrome to all patients [17] [18].

\section{Results}

We reevaluated 70 seronegative and 130 seropositive RA patients. Eighty patients (Group 1) were fulfilling both old and new criteria of fibromyalgia syndrome, and not fulfilling any RA criteria, 65 of them were seronegative and 15

Table 1. Demographic data of study group groups.

\begin{tabular}{cccccccc}
\hline & No & $\%$ & Age & \multicolumn{2}{c}{ Female Sex } & Duration \\
\cline { 5 - 6 } & & & years & N & $\%$ & years \\
\hline Seropositive & 130 & $65 \%$ & $40 \pm 13$ & 130 & $100 \%$ & $6.5 \pm 3.2$ \\
Seronegative & 70 & $35 \%$ & $38 \pm 16$ & 70 & $100 \%$ & $5.9 \pm 2.8$ \\
& 200 & $100 \%$ & & & & \\
\hline
\end{tabular}


were seropositive with low RF titers and negative ACPA. All patients had vitamin D3 deficiency, but it was significantly lower in Group 1. Sixty patients in Group 1 (75\%) had abnormally high levels of PTH $99 \pm 15 \mathrm{pg} / \mathrm{ml}$, and had no parathyroid gland pathology; hence we called them SHPT group (Table 2 and Table 3).

RA (Group 2) were fulfilling either or both ACR and EULAR criteria, and not fulfilling any of Fibromyalgia criteria. They yielded moderate to high scores of DAS 28 (5.68 \pm 1.25$)$, CDAI (30.4 \pm 6.4$)$, and SDAI (31.67 \pm 15.25$)$ (Table 4).

Table 2. Laboratory chemistry and PTH results of both SHPT and RA groups.

\begin{tabular}{ccccccccc}
\hline & No & $\begin{array}{c}\text { ESR } \\
\mathrm{mm}\end{array}$ & $\begin{array}{c}\mathrm{SUA} \\
\mathrm{mg} / \mathrm{dl}\end{array}$ & $\begin{array}{c}\mathrm{Ca} \\
\mathrm{mg} / \mathrm{dl}\end{array}$ & $\begin{array}{c}\mathrm{Ca}^{2+} \\
\mathrm{mg} / \mathrm{dl}\end{array}$ & $\begin{array}{c}\mathrm{D} 3 \\
\mathrm{ng} / \mathrm{ml}\end{array}$ & $\begin{array}{c}\mathrm{PTH} \\
\mathrm{pg} / \mathrm{ml}\end{array}$ & $\begin{array}{c}\mathrm{P} \\
\mathrm{mg} / \mathrm{dl}\end{array}$ \\
\hline Group 1 & & 45 & 7.6 & 8.8 & 1.12 & 12.56 & 99 & 4.9 \\
SHPT & 80 & \pm & \pm & \pm & \pm & \pm & \pm & \pm \\
& & 11 & 2.3 & 1.6 & 0.21 & 4.76 & 15 & 1.8 \\
Group 2 & \multirow{2}{*}{120} & 78 & 5.6 & 9.5 & 2.8 & 14.64 & 45 & 3.8 \\
RA & & 18 & 1.3 & 1.2 & 1.1 & 7.87 & 14 & \pm \\
& & & & & & & & \\
P & & $<0.001$ & $<0.001$ & $<0.001$ & $<0.001$ & $<0.05$ & $<0.001$ & $<0.001$ \\
\hline
\end{tabular}

RA: rheumatoid arthritis, SHPT: secondary hyperparathyroidism, ESR: erythrocyte sedimentation rate, SUA: serum uric acid, Ca: total calcium, $\mathrm{Ca}^{2+}$ : ionized calcium, D3: vitamin D3, PTH: parathyroid hormone, $\mathrm{P}$ : serum phosphorus.

Table 3. Different criteria domains in both SHPT and RA groups.

\begin{tabular}{cccccccccc}
\hline & NO & RN & Dur & CCP & RF & Radiology & TTP & SSS & WPI \\
\hline Group 1 & \multirow{2}{*}{80} & $0 \%$ & \pm & $0 \%$ & $18 \%$ & $0 \%$ & \pm & \pm & \pm \\
SHPT & & & 0.86 & & & & 2.3 & 2.11 & 3.34 \\
& & & 4.6 & & & & 4.2 & 3.11 & 4.31 \\
Group 2 & \multirow{2}{*}{120} & \multirow{2}{*}{$35 \%$} & \pm & $90 \%$ & $70 \%$ & $100 \%$ & \pm & \pm & \pm \\
RA & & & 2.3 & & & & 1.2 & 1.13 & 1.65 \\
P & & $<0.001$ & $<0.001$ & $<0.001$ & $<0.001$ & $<0.001$ & $<0.001$ & $<0.001$ & $<0.001$ \\
\hline
\end{tabular}

RA: rheumatoid arthritis, SHPT: secondary hyperparathyroidism, RN: rheumatoid nodules, Dur: duration, CCP: anti-CCP, RF: rheumatoid factor, TTP: tender trigger points, SSS: symptom severity score, WPI: widespread pain index.

Table 4. Activity scores in both SHPT and RA groups.

\begin{tabular}{|c|c|c|c|c|c|c|c|c|}
\hline & & DAS 28 & SJC & TJC & MS & VAS & CDAI & SDAI \\
\hline \multirow{3}{*}{$\begin{array}{c}\text { Group } 1 \\
\text { SHPT }\end{array}$} & \multirow{3}{*}{80} & 1.61 & 2.01 & 6.54 & & 54.77 & 5.68 & 1.8 \\
\hline & & \pm & \pm & \pm & 0 & \pm & \pm & \pm \\
\hline & & 0.71 & 0.91 & 2.43 & & 11.33 & 1.25 & 0.3 \\
\hline \multirow{3}{*}{$\begin{array}{c}\text { Group } 2 \\
\text { RA }\end{array}$} & \multirow{3}{*}{120} & 5.68 & 7.13 & 11.55 & 31.4 & 63.48 & 30.4 & 31.67 \\
\hline & & \pm & \pm & \pm & \pm & \pm & \pm & \pm \\
\hline & & 1.25 & 5.02 & 7.39 & 29.3 & 25.43 & 6.4 & 15.25 \\
\hline $\mathrm{P}$ & & $<0.001$ & $<0.001$ & $<0.001$ & $<0.001$ & $<0.05$ & $<0.001$ & $<0.001$ \\
\hline
\end{tabular}

RA: rheumatoid arthritis, SHPT: secondary hyperparathyroidism, DAS: disease activity score, SJC: swollen joint count, TJC: tender joint count, MS: morning stiffness, SDAI: simple disease activity index, CDAI: clinicaldisease activity index, VAS: visual analogue scale. 
Plain radiographs of RA (Group 2) show identical RA changes of juxta-articular osteoporosis, subchondral cysts, juxta-articular bony erosions, ulnar deviation of the MCP joints, boutonniere and swan neck deformities, hitchhiker's thumb deformity, and ulnar translocation.

On the other hand, X-rays of SHPT (Group 1) hands showed subperiosteal and subchondral resorption of mainly thumbs, subchondral osteopenia of proximal and middle phalanges, mild subperiosteal resorption along the radial aspect of the middle phalanx and mild tuft erosions, besides changes in the carpus closely resembling those of rheumatoid arthritis, of ulnar styloid resorption, radiocarpal and scapho-trapezoid joint arthritis. Of special interest, the presence of tuft spurs-like excrescences mimicking that of (spade phalanx sign) of acromegaly, but without any other criteria of acromegaly (Figure 1). Plain X ray of knees showed chondrocalcinosis, intracortical resorption, and osteopenia (Figure 2). Lumbar spine shows mild sclerotic bands of the Superior and inferior end plates mimicking rugger jersey sign, as well as osteopenia and brown tumors.

Incidence of X-ray findings of subperiosteal resorption, osteolysis of distal phalanges, brown tumors in lumbar spine, rugger jersey appearance of vertebral column, calcaneal spurs, chondrocalcinosis, and tuft spur-like excrescences were described (Table 5).

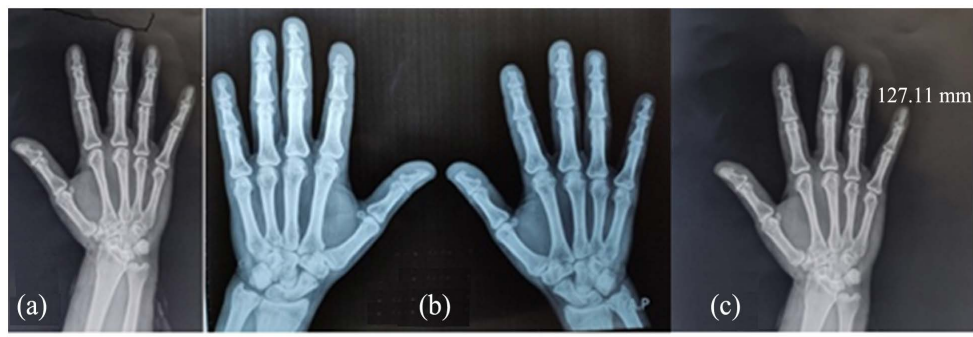

Figure 1. (a) Mild subperiosteal bone resorption affecting radial aspects of the proximal and middle phalanges of the $2 \mathrm{nd}$ and $3 \mathrm{rd}$ fingers. No subligamentous resorption. Terminal tuft erosion (acro-osteolysis), changes in the carpus closely resembling those of rheumatoid arthritis, radiocarpal and scapho-trapezoid joint arthritis. as well as spur-like excrescences bilaterally. ((b), (c)) An AP x ray of the hands showed Superiorsteal and subchondral bone resorption along radial aspect of index, acro osteolysis, as well as spur-like excrescences bilaterally.

Table 5. Different radiological findings in both SHPT and RA groups.

\begin{tabular}{ccccccccc}
\hline Group & N0 & Sr & Odp & Bt & Rj & Cs & ch & Ts \\
\hline $\begin{array}{c}\text { Group 1 } \\
\text { SHPT }\end{array}$ & 80 & $58 \%$ & $8.22 \pm 0.86$ & $23 \%$ & $20 \%$ & $60 \%$ & $5 \%$ & $85 \%$ \\
$\begin{array}{c}\text { Group 2 } \\
\text { RA }\end{array}$ & 120 & $35 \%$ & 0 & 0 & 0 & $5 \%$ & 0 & 0 \\
P & & $<0.001$ & $<0.001$ & $<0.001$ & $<0.001$ & $<0.001$ & $<0.001$ & $<0.001$ \\
\hline
\end{tabular}

RA: rheumatoid arthritis, SHPT: secondary hyperparathyroidism, Sr: Subperiosteal resorption, Odp: Osteolysis of distal phalanges, Bt: Brown tumours, Rj: Rugger jersey appearance of vertebral column, Cs: Calcaneal spurs, ch: chondrocalcinosis, Ts: Tuft spur-like excrescences. 

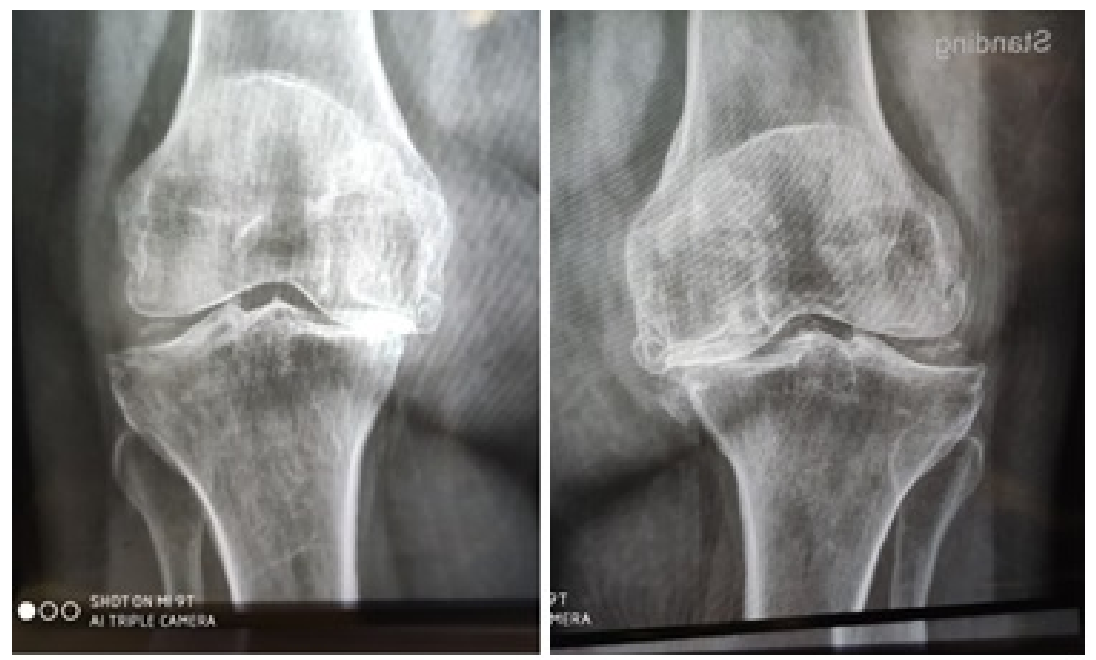

Figure 2. Plain X ray AP knee shows chondrocalcinosis in latral compartment of the knee. Intracortical resorption oval shape and narrow joint space. And bilateral posterior loose bodies. Subperiosteal bone resorption medial aspect femur.

Patients who reevaluated as having Fibromyalgia/SHPT syndrome stopped RA treatment, and were allocated to fibromyalgia regimen of SSRI, muscle relaxants, and vitamin D3 supplementation 5000 - 10,000 iu/day, in addition to sulphasalazine $1-2 \mathrm{gm} /$ day to which their inflamed joints responded significantly.

\section{Discussion}

We found many patients with bilateral wrists and hands arthritis who had a constellation of vitamin D3 deficiency, elevated parathyroid hormone, and fibromyalgia. They were misdiagnosed as either seronegative or seropositive rheumatoid arthritis, which resulted in nonresponse to treatment and permanent discomfort. A study by Helliwell showed arthritis in $11 \%$ of HPT patients, including two cases of erosive arthritis [19]. HPT presenting as arthritis had been described in few case reports [19] [20] [21] [22] [23]. Meanwhile, HPT can occasionally co-exist with inflammatory arthritis like rheumatoid arthritis [24].

Radiographical features were helpful to differentiate both, in addition to clinical and biochemical features. Other than HPT causes osteoporosis and pathological fractures,it can cause bone erosions, which are mostly shaggy in appearance and distributed in the radiocarpal, radioulnar, metacarpophalangeal and distal interphalangeal joints, in contrast to that which predominate proximal interphalangeal joints of rheumatoid arthritis [25]. HPT is a disease of increased bone resorption and bone formation. Subsequently, plain radiographic findings may include resorption and sclerosis of numerous sites in the skeletal system [26]. Historically, osteitis fibrosa cystica was used to describe the advanced skeletal disease in primary hyperparathyroidism. Bone findings were characterized by the osteoclastic resorption of bone, osteoblastic bone formation, and fibrous replacement of marrow, with radiographic findings of subperiosteal resorption, 
brown tumors, bone cysts, and sclerosis [27].

Nowadays, the most common radiologic finding in HPT is osteopenia, which may be generalized or asymmetric. Fine trabeculations are initially lost, with resultant coarse and thickened trabeculae. The disease may progress with further destruction that results in a ground-glass appearance in the trabeculae [27]. Other methods for the quantification of bone density, such as QCT scanning and DXA, are more sensitive in the evaluation of osteopenia [27]. Additional findings include bone resorption, which may occur at many different anatomic sites. Bone resorption may be classified as subperiosteal, intracortical, trabecular, endosteal, subchondral, subligamentous, or subtendinous. Subperiosteal bone resorption is an early and virtually pathognomonic sign of HPT, which is marked by marginal erosions with adjacent resorption of bone and sclerosis. An unusual lacelike appearance may be seen beneath the periosteum with an occasional spiculated external cortex. The underlying resorptive process may progress to complete cortical disappearance [27]. Although subperiosteal bone resorption can affect many sites, the most common site in HPT is the middle phalanges of the index and middle fingers, primarily on the radial aspect [27].

Of special interest, the presence of tuft spurs-like excrescences mimicking that of spade phalanx sign of acromegaly [28], but without any other criteria of acromegaly. A sole literature reported this sign in an unexplained case report, [29].

Due to lack of typical clinical features, hyperparathyroidism is often undiagnosed or misdiagnosed, especially in unspecialized centers. Hence, rheumatologists should have a high degree of suspicion for differential diagnosis at the beginning and during follow-ups especially when the initial diagnosis is doubtful, or the treatment is not effective.

\section{Conclusion}

We have characterized a syndrome of VitaminD3 deficiency/Fibromyalgia/ Hyperparathyroidism mimicking Rheumatoid Arthritis. It is characterized by longstanding vitamin D3 deficiency, associated with hyperparathyroidism and fibromyalgia, predominantly negative Anti CCP, while RF may be low positive. Radiologically is characterized by the presence of finger tuft acro-osteolysis with spur like excrescences which is pathognomonic, besides other features of hyperparathyroidism including subperiosteal and subchondral resorption of mainly thumbs, subchondral osteopenia of proximal and middle phalanges, mild subperiosteal resorption along the radial aspect of the middle phalanx and mild tuft erosions, besides changes in the carpus closely resembling those of rheumatoid arthritis, of ulnar styloid resorption, radiocarpal and scapho-trapezoid joint arthritis. This syndrome responds well to treatment of fibromyalgia regimen of SSRI, muscle relaxants, and vitamin D3 supplementation 5000 - 10,000 iu/day, in addition to sulphasalazine 1 - $2 \mathrm{gm} /$ day to which their inflamed joints responded significantly. 


\section{Conflicts of Interest}

All authors confirm that there are no any potential conflicts of interest of each of them. We confirm hereby that the manuscript has not been submitted or is not simultaneously being submitted elsewhere, is not at the time of submission under consideration by another journal or other publication, and that no portion of the data has been or will be published elsewhere while the manuscript is under review by the journal, unless rejected or withdrawn by the author. Also we confirm that no portion of the data has been or will be published elsewhere while the manuscript is under review by the journal.

\section{Financial Support}

We confirm also that there are not any financial support or other benefits from commercial sources for the work reported on in the manuscript, or any other financial interests that any of the authors may have, which could create a potential conflict of interest or the appearance of a conflict of interest with regard to the work.

\section{References}

[1] Taylor, J.G. and Bushinsky, D.A. (2009) Calcium and Phosphorus Homeostasis. Blood Purification, 27, 387-394. https://doi.org/10.1159/000209740

[2] Pepe, J., Cipriani, C., Pilotto, R., De Lucia, F., Castro, C., Lenge, L., et al. (2011) Sporadic and Hereditary Primary Hyperparathyroidism. Journal of Endocrinological Investigation, 34, 40-44.

[3] Madkhali, T., Alhefdhi, A., Chen, H. and Elfenbein, D. (2016) Primary Hyperparathyroidism. Ulusal Cerrahi Dergisi, 32, 58-66.

[4] Falkenburg, W.J.J. (2015) IgG Subclass Specificity Discriminates Restricted IgM Rheumatoid Factor Responses from More Mature Anti-Citrullinated Protein Antibody-Associated or Isotype-Switched IgA Responses. Arthritis \& Rheumatology, 67, 3124-3134. https://doi.org/10.1002/art.39299

[5] Edkins, A. and Cushley, W. (2012) The Jekyll and Hyde Nature of Antibodies. Biological Sciences Review, 25, 4.

[6] Vossenaar, E.R., Smeets, T.J., Kraan, M.C., Raats, J.M., van Venrooij, W.J. and Tak, P.P. (2004) The Presence of Citrullinated Proteins Is Not Specific for Rheumatoid Synovial Tissue. Arthritis \& Rheumatology, 50, 3485-3494.

https://doi.org/10.1002/art.20584

[7] Elbeialy, A. and Eldesouky, H. (2019) AB1261 Cadmium Toxicity as a Probable Cause of Osteopenia in Adolescents and Its Relation to Bad Dietary Habits. 2091-2092. https://doi.org/10.1136/annrheumdis-2019-eular.731

[8] Kazantzis, G. (2004) Cadmium, Osteoporosis and Calcium Metabolism. BioMetals, 17, 493-498. https://doi.org/10.1023/B:BIOM.0000045727.76054.f3

[9] Wang, Y., Zhu, J. and DeLuca, H.F. (2015) The Vitamin D Receptor in the Proximal Renal Tubule Is a Key Regulator of Serum 1 $\alpha, 25$-Dihydroxyvitamin $\mathrm{D}_{3}$. The American Journal of Physiology-Endocrinology and Metabolism, 308, E201-E205. https://doi.org/10.1152/ajpendo.00422.2014

[10] Kurata, Y., Katsuta, O., Doi, T., et al. (2014) Chronic Cadmium Treatment Induces Tubular Nephropathy and Osteomalacia Osteopenia in Ovariectomized Cynomol- 
gus Monkeys. Veterinary Pathology, 51, 919-931.

https://doi.org/10.1177/0300985813509384

[11] Costa, J.M., Ranzolin, A., Costa Neto, C.A., Marques, C.D. and Duarte, A.L. (2016) High Frequency of Asymptomatic Hyperparathyroidism in Patients with Fibromyalgia: Random Association or Misdiagnosis? Revista Brasileira de Reumatologia, 56, 391-397. https://doi.org/10.1016/j.rbre.2016.03.008

[12] Borgia, A.R., Cavallasca, J.A., Costa, C.A. and Musuruana, J.L. (2012) Hyperparathyroidism, a Forgotten Cause of Musculoskeletal Pain. Reumatología Clínica, 8, 299-301. https://doi.org/10.1016/j.reumae.2012.06.019

[13] Mackenzie-Feder, J., Sirrs, S., Anderson, D., Sharif, J. and Khan, A. (2011) Primary Hyperparathyroidism: An Overview. International Journal of Endocrinology, 2011, Article ID: 251410.

[14] Arnett, F.C., Edworthy, S.M., Bloch, D.A., McShane, D.J., Fries, J.F., Cooper, N.S., et al. (1988) The American Rheumatism Association 1987 Revised Criteria for the Classification of Rheumatoid Arthritis. Arthritis \& Rheumatology, 31, 315-324. https://doi.org/10.1002/art.1780310302

[15] Aletaha, D., Neogi, T., Silman, A.J., Funovits, J., Felson, D.T., Bingham, C.O., et al. (2010) 2010 Rheumatoid Arthritis Classification Criteria: An American College of Rheumatology/European League against Rheumatism Collaborative Initiative. Arthritis \& Rheumatology, 62, 2569-2581. https://doi.org/10.1002/art.27584

[16] Smolen, J.S. and Aletaha, D. (2014) Scores for All Seasons: SDAI and CDAI. Clinical and Experimental Rheumatology, 32, S-75-9.

[17] Wolfe, F., Smythe, H.A., Yunus, M.B., Bennett, R.M., Bombardier, C., Goldenberg, D.L., et al. (1990) The American College of Rheumatology 1990 Criteria for the Classification of Fibromyalgia. Report of the Multicenter Criteria Committee. Arthritis \& Rheumatology, 33, 160-172.

[18] Wolfe, F., Clauw, D.J., Fitzcharles, M.A., Goldenberg, D.L., Katz, R.S., Mease, P., et al. (2010) The American College of Rheumatology Preliminary Diagnostic Criteria for Fibromyalgia and Measurement of Symptom Severity. Arthritis Care \& Research, 62, 600-610. https://doi.org/10.1002/acr.20140

[19] Helliwell, M. (1983) Rheumatic Symptoms in Primary Hyperparathyroidism. Postgraduate Medical Journal, 59, 236-240. https://doi.org/10.1136/pgmj.59.690.236

[20] Bywaters, E.G.L., Dixon, A.S.J. and Scott, J.T. (1963) Joint Lesions of Hyperparathyroidism. Annals of the Rheumatic Diseases, 22, 171-187. https://doi.org/10.1136/ard.22.3.171

[21] Zvaifler, N.J., Reefe, W.E. and Black, R.L. (1962) Articular Manifestations in Primary Hyperparathyroidism. Arthritis \& Rheumatology, 5, 237-249.

https://doi.org/10.1002/art.1780050304

[22] Lipson, R.L., Williams, L.E., Zvaifler, N.J. and Howell, D.S. (1968) The "Connective Tissue Disorder” of Hyperparathyroidism. Arthritis \& Rheumatism: Official Journal of the American College of Rheumatology, 11, 198-205. https://doi.org/10.1002/art.1780110211

[23] Duraywish, A.A. (2016) Chronic Unclassified Polyarthritis: A Rare Presentation of Primary Hyperparathyroidism. The Medical Journal of Cairo University, 84, 265-269.

[24] Salari, M., Aboutorabi, R.B. and Rezaieyazdi, Z. (2015) Hypercalcemia and Lytic Bone Lesions Masquerading Inflammatory Arthritis Treated as Rheumatoid Arthritis. Archives of Iranian Medicine, 18, 720-723.

[25] Feng, X. and McDonald, J.M. (2011) Disorders of Bone Remodeling. Annual Review 
of Pathology, 6, 121-145. https://doi.org/10.1146/annurev-pathol-011110-130203

[26] Li, N., Li, X.M., Xu, L., Sun, W.J., Cheng, X.G. and Tian, W. (2013) Comparison of QCT and DXA: Osteoporosis Detection Rates in Postmenopausal Women. International Journal of Endocrinology, 2013, Article ID: 895474.

https://doi.org/10.1155/2013/895474

[27] Patel, A.A., Ramanathan, R., Kuban, J. and Willis, M.H. (2015) Imaging Findings and Evaluation of Metabolic Bone Disease. Advances in Radiology, 2015, Article ID: 812794. https://doi.org/10.1155/2015/812794

[28] Killinger, Z., Payer, J., Lazúrová, I., Imrich, R., Homérová, Z., Kužma, M. and Rovenský, J. (2010) Arthropathy in Acromegaly. Rheumatic Disease Clinics of North America, 36, 713-720. https://doi.org/10.1016/j.rdc.2010.09.004

[29] Freyschmidt, J., Brossmann, J., Sternberg, A., et al. (2003) Freyschmidt's “Koehler/Zimmer's Borderlands of Normal and Early Pathological Findings in Skeletal Radiography". 